\title{
Simulation fragmentation of samples of rock at explosive loading
}

\author{
Vitaly Trofimov ${ }^{1}$ and Ivan Shipovskii ${ }^{*}$ \\ ${ }^{1}$ Institute of Comprehensive Exploitation of Mineral Resources, Russian Academy of Sciences, \\ 111020, Moscow, Russia
}

\begin{abstract}
Research to improve the definition of rational parameters for blasting is becoming increasingly important and valuable. This is especially true in the era of digital technology, which allows miners to realize a holistic vision of optimizing the entire process - from the quarry to the receipt of the final product at the processing plant. The proposed computer simulation based on the Smoothed Particle Hydrodynamics (SPH) method in AUTODYN computer complex to optimize the explosion results by integrating the initial data - charge parameters and rock properties, is aimed at improving measures that reduce the total cost of drilling and blasting, increasing mining productivity and safety. The presented calculations make it possible to estimate the number of fragments and their initial expansion velocity for various explosives. Key words: explosive, elongated charge, fragmentation, computer simulation, meshless SPH code.
\end{abstract}

\section{Introduction}

In the mining industry, the gran composition of the rock mass, separated from the massive, is used to evaluate the results of blasting operations (especially the oversized yield) and is taken into account when choosing the type and parameters of technological equipment in quarries. Of particular interest to mining enterprises is the percentage of oversized fraction output, because the output and the life of the loading and transport equipment and the reliability of its operation depend on the oversized fraction. The time spent on secondary crushing violates the rhythm of the enterprise, as well as lead to additional consumption of explosives and means of initiation.

In such conditions, the search for ways to improve the quality of preparation of blasted rock mass and the stability of obtaining the required grain size of rocks, ensuring the rhythmic operation of loading equipment and mining equipment is very important. In this regard, in this work, the process of destruction of a sample from a rock by a blast is investigated by a numerical simulation.

\footnotetext{
* Corresponding author: shipovskiy i@ipkonran.ru
} 


\section{Theory}

To evaluation the kinetics of fragmentation and physical indicators of the effect of the explosion on destructible geomedia, a computer simulation was carried out using a numerical model [1] (fig. 1-b), which according to geometric and physical properties corresponds to an experimental simulation physical model (fig. 1-a) [2].

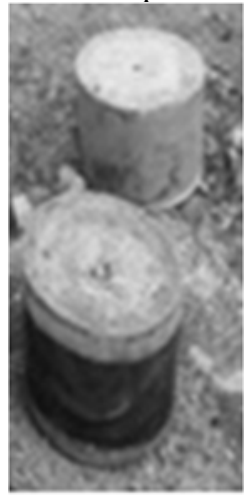

a

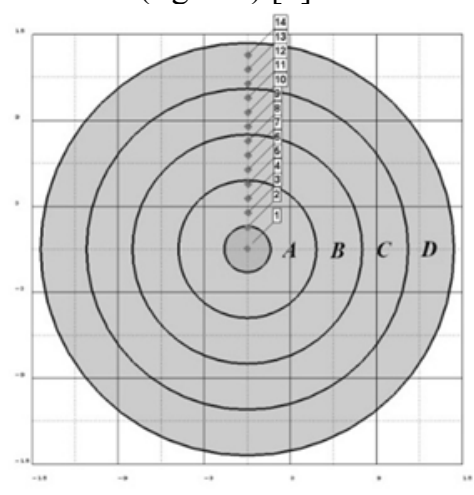

b

Fig. 1. Experimental samples (a) and computer model (b)

In figure $1 \mathrm{~b}: A, B, C, D$ - conventional zones of the rock sample; numbers inside the frames - places of conditional numerical sensors for recording changes in process parameters; the ANFO explosive charge is placed in the center of the sample; the detonator is located in the center of the charge.

The computer simulation results of deformation and the sample fracture under the influence of explosive loads were recorded at various time points with visualization in the form of graphs and chronograms of fracture and expansion of fragments.

To describe the fragmentation in this paper, after analyzing the advantages and disadvantages of various analytical and numerical approaches, the Smoothed Particle Hydrodynamics (SPH) method was chosen [1]. As the practice of calculations showed [1, 3-7], this numerical method automatically takes into account a wide range of physical phenomena related to the nonlinearity of the loading process and possible violation of rock continuity.

The objectives of the research presented in this paper can be formulated as follows:

- conduct computer simulation of the fragmentation of the explosive sample with varying characteristics of the explosive;

- determine, on the basis of numerical experiments, the parameters of the consequences of fragmentation: velocities, characteristic size and mass distribution of the fragments of the sample formed during the explosion;

- to trace the change in the behavior of some parameters that are practically immeasurable in practice, such as stress, strain rate, and other characteristics of the stressstrain state of a sample in characteristic zones.

To formulate the problem and solve it, the complete system of equations for mechanics of a deformable solid is used, including the equation of motion, the continuity equation, the energy balance equation, the Cauchy relations, the equation of state (describing the spherical part of the stress tensor), and the defining equations (showing the relationship between the stress deviators and deformations). This system should be closed by relations defining changes in the quantities included in the basic equations as parameters.

In describing explosives and adiabatic expansion of detonation products, the Jones Wilkins - Lee equation of state (JWL) was adopted [1]. This equation represents pressure as a function 


$$
p(\rho, e)=A\left(1-\frac{\omega \eta}{R_{1}}\right) e^{-R_{1} / \eta}+B\left(1-\frac{\omega \eta}{R_{2}}\right) e^{-R_{2} / \eta}+e \omega \eta,
$$

where $\eta=\rho / \rho_{e}$ is the ratio of the density of detonation products to the explosive charge density, $e$ is the specific detonation energy $A, R_{1}, B, R_{2}, \omega$ are constants whose values for the explosives used are determined from explosive experiments by comparing the results numerical calculations of the functioning of various explosive devices with experimental data.

The deformation and strength behavior of the geomaterial is evaluated by the DruckerPrager criterion, according to which, when the elastic limit is reached, a transition to plastic flow occurs. In this case, the elastic behavior of the geomaterial follows Hooke's law, which defines a linear relationship between the velocity deviator of the stress tensor and the strain rate tensor. The elastic state of the medium in the stress space is limited by the surface of the limiting state, upon reaching which the process of inelastic, plastic deformation, or fracture begins.

$$
f\left(\sigma_{i j}, \varepsilon_{i j}^{p}\right)=0
$$

where $f$ is the equation of the loading surface, , $\sigma_{i j}$ is the stress tensor, $\varepsilon_{i j}^{p}$ is the plastic strain tensor. Plastic deformation is determined from the equation:

$$
d \varepsilon_{i j}^{p}=d \lambda \frac{\partial g}{\partial \sigma_{i j}},
$$

where $g\left(\sigma_{i j}, \varepsilon_{i j}^{p}\right)$ is the plastic potential $d \lambda$ is determined in the course of deformation from the condition of plasticity:

$$
\left(\sigma_{1}-\sigma_{2}\right)^{2}+\left(\sigma_{1}-\sigma_{2}\right)^{2}+\left(\sigma_{1}-\sigma_{2}\right)^{2}=2 Y^{2},
$$

where $\sigma_{1}, \sigma_{2}, \sigma_{3}$ - principal stresses, $Y$ - elastic limit for simple tension.

In numerical modeling, it is believed that for the selected model geometry (fig. 1-b), due to the extension of the elongated charge, the conditions of plane deformation are realized and the solution of the problem can be obtained in $2 D$ geometry.

Further, to close the system of equations by setting the initial and boundary conditions, a boundary-value problem was formulated. For this, the outer surface of the cylindrical model is without stress and displacement and on the surface of the well to place the charge, the pressure of the explosion products was set, which is obtained in the framework of solving the same problem, taking into account the detonation of the explosive (fig. 2).

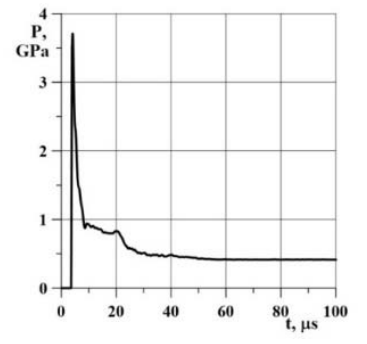

a

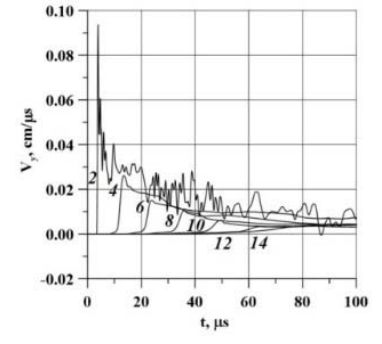

b

Fig. 2. a) - The calculated pressure pulse of the detonation products of the explosive charge on the well wall; b) is the rock velocity of the specimen indicated in fig. 1-b markers.

The change in the strength of the loaded medium over time is taken into account by introducing the function of damage accumulation

$$
D\left(\sigma_{i j}, t\right)=\int \frac{\left(S-S_{0}\right)^{n}}{\left(S^{*}\right)^{n} t^{*}} d t, \text { for } S>S_{0},
$$

where $S$ - effective stress; $S_{0}=250 \mathrm{MPa}, S^{*}, t^{*}, n$ - parameters determining the threshold stress, starting from which damage is accumulated, ultimate stress and parameters characterizing the rate of damage accumulation. In this case, it is assumed that the 
weakening, softening, and disengagement, as a result of damage, are functionally set through the modules of the geomedium

$$
K=\left(1-D^{2}\right) K_{0}, \mu=\left(1-D^{2}\right) \mu_{0}, \quad Y=\left(1-D^{2}\right) Y_{0} .
$$

That is, the bulk modulus is reduced $K$, shear modulus $\mu$ and yield strength $Y$ from their base values for intact material $K_{0}, \mu_{0}$ and yield strength $Y_{0}$, respectively. The components of compressive stresses do not decrease as the fracture occurs, since the material in the fracture region remains capable of transmitting compressive forces.

\section{Calculation results}

The figure 3 shows a photograph of a simulation composite model (a), destroyed by the explosion energy of the sparing action of a granular explosive based on ANFO ammonium nitrate [2], as well as a calculated image (b) of a numerical model destroyed by the explosion energy.

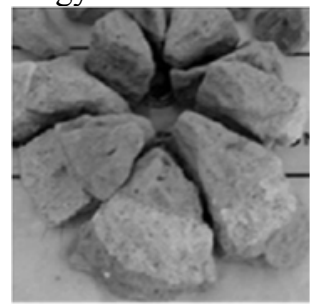

a

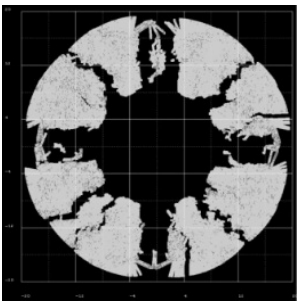

b

Fig. 3. The experimental sample (a) and the computer model (b)

A comparison of these images shows a fairly close similarity in the nature of the formation of fragments, in particular in the number of large pieces. Detonation products create an intense compression wave. The compression wave provides primary crushing, and the subsequent crushing stage is mainly due to the unloading of the rock after the blast wave reaches the free surface and the restructuring of the velocity field (fig. 4).

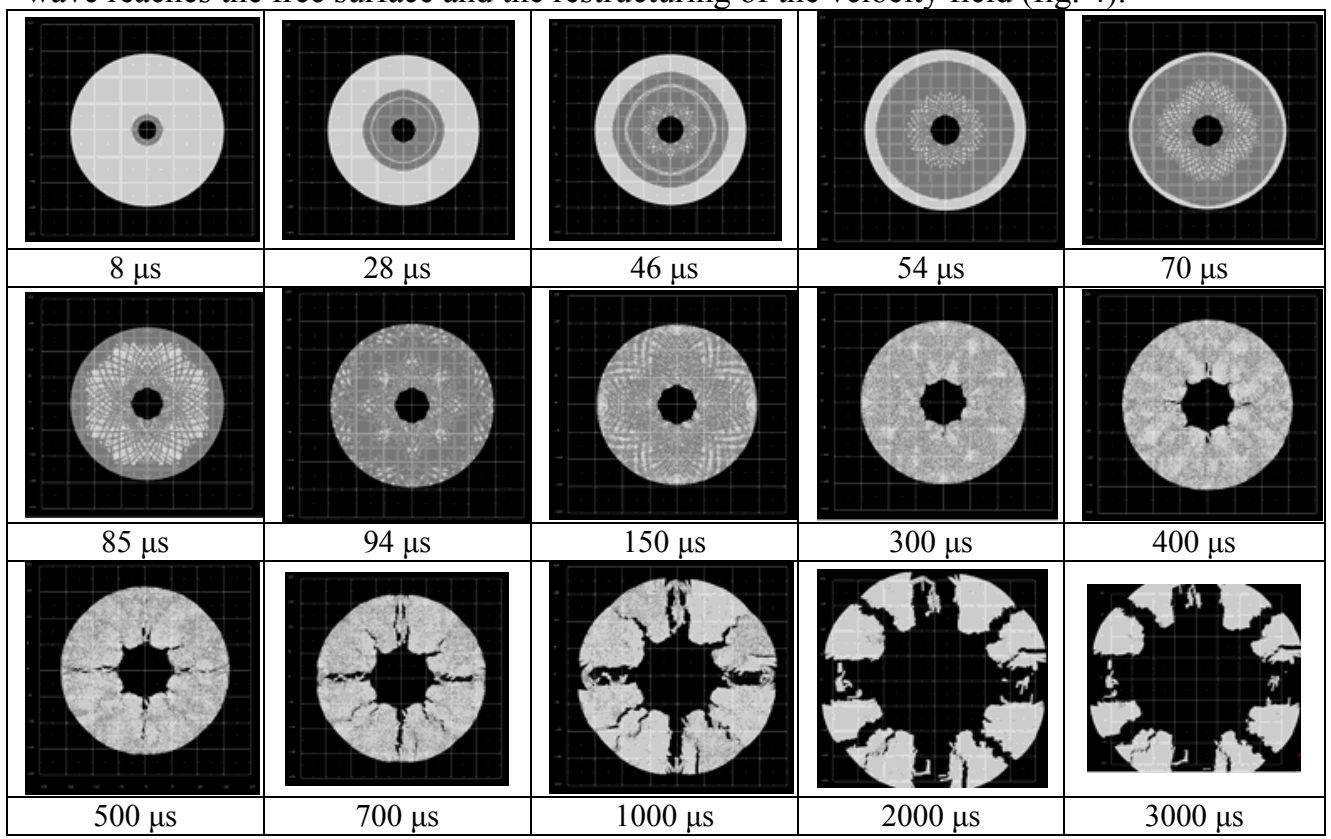

Fig. 4. A chronogram of the localization of deformations and the development of fractures in the sample when the borehole blast. 
Figure 4 shows a chronogram of the development process of the sample material deformations under the influence of detonation products of the explosive charge.

The processes of deformation of geomaterials are accompanied by the observed localization of shifts. Localization occurs with the movement of rocks around the wells. This phenomenon is universal in nature and is noted in metals and other plastic materials. Localization shows a significant effect on all parameters of the stress-strain state of the geomedium. Without taking it into account, it is impossible to carry out an adequate calculation of either kinematics of deformation or fracture processes.

The formation and development of localization zones (fig. 4. 46-300 $\mu$ s) similar to the formation of cracks in solids. In both cases, we are talking about surfaces with a strong discontinuity of displacements. Moreover, the specifics of the deformation of the geomedium is associated with the presence of large compressive stresses. Therefore, the bearing capacity of the medium after localization is not exhausted, but a new stage of deformation occurs (fig. 4. 400-700 $\mu$ s). The formation of a grid of slip lines in the form of intersecting logarithmic spirals is clearly traced (fig. 4.70-150 $\mu$ s). The obtained pictures confirm the adequacy of the calculation results to the classical mathematical models of the plastic behavior of the geomedium.

During the propagation of localized shear bands due to dilatancy, a proppant may occur, resulting in the formation of separation cracks propagating in the sample (fig. 4. 400$700 \mu \mathrm{s})$. The formation of bands of localized compaction occurs at the initial stage of compaction, when a change in the stress-strain state is accompanied by the smallest expansion of the surface of the limiting state. As the load increases, the zone of localized compaction gradually expands to the entire sample, after which the degree of compaction in the sample is leveled and subsequently proceeds uniformly (fig. 4. 300-500 $\mu \mathrm{s}$ ).

The formation of localization bands is associated with loading of close zones of the medium along different paths, which may be due to the heterogeneity of the stress-strain state and dynamic disturbance of the stress state. With the beginning of localization of the deformation of the loading path of points lying in the localization zone and outside it, they sharply change their direction. Moreover, during the formation of compression and shear bands in the process of compaction, the directions of changes in the loading paths are opposite. The formation of a periodic system of localized deformation bands under the conditions of shear deformation of a geomedium layer is a characteristic feature of the nonlinear behavior of media with internal friction and dilatancy. The orientation of these bands is determined, first of all, by the nature of loading and the properties of the medium, while the geometry of the region significantly affects their periodicity. A change in the stress state leads to a change in the deformation mode. (fig. 4. 700-3000 $\mu$ s)

In explosions in rock-type samples, the fracture zone is divided into two areas: a crushing (crushing) zone, where the rock turns into a finely divided mass with a crushed structure, and a loosening zone with radial tangential cracks. After the passage of the blast wave, a certain destructive effect in this zone is also produced by the explosion gases under high pressure. The rock near the charge under the influence of the blast wave (fig. 2-a) and gases is rapidly compressed and displaced after the front of the stress wave. As a result, it is strongly deformed, a system of numerous cracks is formed in the rock. As you move away from the charge, the stresses in the rock from the explosion decrease and at a certain distance become less than the resistance of the rock to compression, due to which the nature of the deformation and destruction of the medium changes.

Under the action of a wave of stresses and compressed explosion gases propagating from the charge in the medium in the radial direction, compressive stresses arise, and tensile stresses arise in the tangential direction (fig. 5.). 


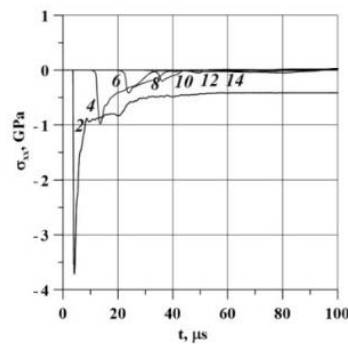

a

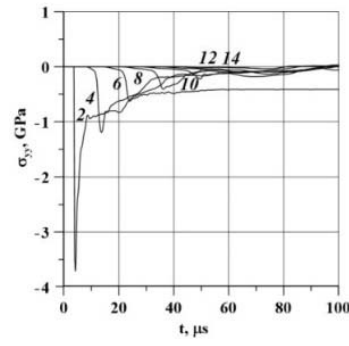

b

Fig. 5. The numerical values and kinetics of the tangential (a) and radial stresses (b) recorded in the sensors marked in fig. $1 \mathrm{~b}$.

From the graphs in fig. 5 shows that by the time moment of $50 \mu$ s the nature of the change in radial and tangential stresses in time is the same. By this time, the sample is unloaded to values whose values for the used numerical model are not significant.

Due to the action of tensile stresses, radial cracks form in the rock (fig. 4. 400-700 $\mu$ s). A certain effect on the opening of these cracks is also exerted by the flow of expanding gases rushing into the cracks. The flow rate can be quite high, and in this case, the gas reaches the top of the crack. Since the gas flow in a narrow crack is associated with noticeable hydrodynamic and heat losses, its pressure in the crack rapidly decreases and becomes insufficient for rupture, but continues to play the role of a lever or wedge, increasing tensile stresses at the crack tip.

This effect is of great importance for the onset of fragmentation. In fig. 6 circle symbols show the calculation of the growth of the number of fragments generated by the energy of the explosion of explosives charges blasting explosive ANFO versus time.

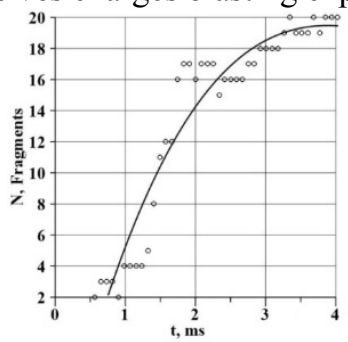

Fig. 6. Graph of the time variation of the calculated total number of sample fragments when exposed to explosive energy using a blasting explosive using ANFO.

At the same time, the onset of fragmentation of the sample is observed after $0.5 \mathrm{~ms}$. The dependence of the growth in the number of fragments under the influence of explosion energy in the initial period of time is nonlinear polynomial in nature ( 3 order) on time. Obviously, at large times this curve reaches a horizontal asymptote due to the attenuation of the process and the limited number of fragments.

We can assume that practically this happens at $t=4 \mathrm{~ms}$, when the crushing of the material basically ends. At this point, many small fragments are formed, the general weight parameters of which are given in Table 1 (in grams).

Table 1. Small Particle summary

\begin{tabular}{|c|c|c|c|c|}
\hline Total number & Total mass & Lowest Mass & Average Mass & Largest Mass \\
\hline 3156 & 7.3460 & 0.002309 & 0.002327 & 0.002327 \\
\hline
\end{tabular}


In addition, 20 fragments are formed, which can be attributed to large ones. Fig 7 shows their weight distribution, ranging from $0.1 \mathrm{~g}$ to $\sim 200 \mathrm{~g}$. In this case, 8 fragments have almost the same weight. They are clearly visible in figures $3 \mathrm{~b}$ and 4 .

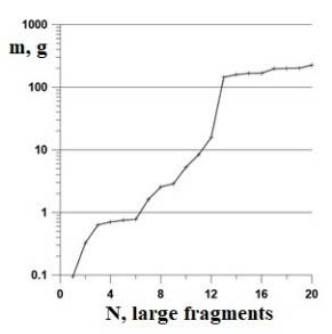

a

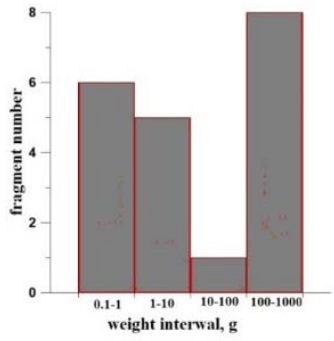

b

Fig. 7. Weight parameters of 20 large fragments (a) and their distribution by weight (b).

\section{Conclusions}

Thus, in the explosion of a borehole elongated charge in a rock sample in the immediate vicinity of the charge, the geomedium is destroyed into a significant number of pieces under the influence of waves of stress and pressure of the explosion gases. By changing the parameters of the explosive charge, it is possible to directionally change the crushing size of the rock in this zone. The sizes of the controlled crushing zone increase discretely with increasing charge diameter, i.e. one, two, three, destructible fragments.

Outside the controlled crushing zone, the destruction of the fragments composing the remaining volume of the exploded sample occurs as a result of the dynamic effect of the expanding volume of the rock on it. The destruction of the rock in the zone of practically unregulated crushing is probabilistic in nature: a fragment can collapse into a small number of parts depending on the charge power as a result of dynamic impact of adjacent sections of rocks. If a fragment has defects or non-uniformities, the probability of destruction increases. A fragment without defects in a zone of practically unregulated crushing, as a rule, is not destroyed.

The object of crushing control methods is to increase the volume of the controlled crushing zone in one way or another, to reduce or completely eliminate the zone of practically unregulated crushing, to crush oversized fragments in this zone.

The work was carried out with the financial support of the RFBR grant 18-05-00912.

\section{References}

1. Explosion Physics / L.P. Orlenko, (Ed.), 2 (2004)

2. $\quad$ N.N. Efremovtsev, Explosion technology, 113(70) (2015)

2. R.A. Bakeev, Yu.P. Stefanov, A.A. Duchkov, and A.V. Myasnikov, AIP Conference Proceedings 1909 (2017)

3. V.N. Zakharov, V. A. Trofimov, I.E. Shipovskii, O.N. Malinnikova, Izvestiya Tula State University, 1 (2020)

4. I. E. Shipovskii, Scientific Bulletin of National Mining University, 1 (145) (2015)

5. V.N. Odintsev, I.E. Shipovskii, EPJ Web of Conferences 221 (2019)

6 V.N. Odintsev, I.E. Shipovskii, Journal of Mining Science, 55(4) (2019)

7. V.A. Trofimov, I.E. Shipovskii, O.N. Malinnikova, Wen-Jie Xu, AIP Conference Proceedings, 10.1063/1.5132196 (2019) 